\title{
Fibroin Secretion in the Posterior Silk Gland Cells of a Flimsy Cocoon Mutant of Bombyx mori
}

\author{
Noriko Adachi-Yamashita*, Bungo Sakaguchi and Haruo Chikushi \\ Laboratory of Sericulture, Faculty of Agriculture, Kyushu University, Hakozaki, \\ Higashi-ku, Fukuoka 812, Japan
}

\begin{abstract}
Developmental changes in the fine structure of the posterior silk gland cells were studied with a recessive mutant of Bombyx mori named flimsy cocoon $(f c)$ whose cocoon-shell weight is much less than that of the normal strain of $B$. mori. The $f c$ posterior silk gland develops normally until $60 \mathrm{~h}$ after the fourth ecdysis. By the 72nd hour, unusual numbers of globules, which we have called as fibroin globules, begin to accumulate in the apical cytoplasm of the mutant cell. These globules increase in number until the 96th hour, when they fill most of the apical cytoplasm and extend into the basal region. In the normal cell at the corresponding stages, massive amounts of fibroin are synthesized and secreted rapidly with no storage of fibroin globules.
\end{abstract}

The silk gland of the silkworm, Bombyx mori, synthesizes large quantities of the fibrous proteins fibroin and sericin, especially during the final larval instar. Fibroin in synthesized in the posterior silk gland then secreted into the lumen of the gland. From there it flows to the middle silk gland where sericin is secreted. The two silk proteins are stored in the lumen in a gelatinous state until they are spun into silk fiber to form the cocoon-shell around the larva.

The weight of cocoon-shells vary with the strain and are affected greatly by physiological and environmental factors. Therefore it has proved difficult to isolate mutants of silk production which show single factor Mendelian inheritance. Despite these difficulties, some mutants have been recovered and propagated in the laboratory. One example is the flimsy cocoon mutant $(f c)$ (3), in which the cocoon-shell is thin $(30 \%$ the weight of a normal cocoon), and the amounts of secreted proteins, especially fibroin, are small. General impairment is slight in this mutant: even the silk gland grows normally till the early fifth instar ( $0-72 \mathrm{~h}$ after the fourth ecdysis), and in these young silk glands no difference was observed between $f l c$ and normal under a light microscope. However, in the mature larva (shortly before spinning) the gland is significantly lighter due to the reduced amounts of gelatinous silk proteins stored in the lumen. The specific activity of $\left[{ }^{3} \mathrm{H}\right]$ glycine incorporated into the TCA-precipitable proteins of the whole fraction of the $f l c$ posterior silk gland does not increase after the 72nd hour of the fifth instar, but that of the normal silk gland continues to increase till the 120th hour (1). Thus, we assumed that some step in the biosynthesis and/or secretion of fibroin in the $f l c$ posterior silk gland is impaired by the action of the

* Present address: Junshin Women's Junior College, 1-1-1 Chikushigaoka, Minami-ku, Fukuoka 810, Japan.

Abbreviations: TCA, trichloroacetic acid. ER, endoplasmic reticulum 
mutant gene, and made a morphological study of the $f l c$ cell with an electron microscope.

The strain of $B$. mori used was the b-60 stock. This is maintained at the Institute of Silkworm Genetics, Faculty of Agriculture, Kyushu University by crossing $\mathrm{Ze}+/+f l c$ females to $+f l c /+f l c$ males. Larvae that are homozygous and heterozygous for $f c$ appeared in a $1: 1$ ratio in the $F_{1}$. Heterozygotes were used as controls. Larvae of this strain, bred on mulberry leaves, matured about $120 \mathrm{~h}$ after the fourth ecdysis and began to spin.

Their posterior silk glands were dissected and washed with physiological saline. Segments of a middle portion were fixed for several hours in cold $2.5 \%$ glutaraldehyde in $0.1 \mathrm{M}$ cacodylate buffer, $\mathrm{pH} 7.4$, containing $0.25 \mathrm{M}$ sucrose, then fixed with $1 \%$ $\mathrm{OsO}_{4}$ in Palade's veronal acetate buffer, $\mathrm{pH}$ 7.4. After fixation the organ segments were dehydrated with ethanol and embedded in Epon 812. These blocks usually were stained with uranyl acetate dissolved in alcohol. After ultrathin sectioning with a Porter-Blum Sorvall MT-1 ultramicrotome, the specimens were doubly stained with uranyl acetate and lead mixture. The electron microscope used was JEM T-8.

It was not until the 72nd hour after the fourth ecdysis that ultrastructural differences between the $f c$ and normal posterior silk gland cells became obvious. In normal cells, lamellar ER predominates in the young silk gland (60th hour) but by the 96th hour (Fig. 1A) this was replaced by the tubular or vesicular ER. We assume that this transformation of ER accompanies fibroin synthesis (7). In the $f l c$ cell, rough ER elements develop normally until the 60th hour, but at the 96th hour these are observed only in the narrow spaces between globules (Fig. 1B), and lamellar ER is not fully transformed to tubular or vesicular ER. In the normal cell, the mitochondria are long and plentiful, but in $f l c$ they are mainly circular in profile and are present in small numbers. The number and size of the Golgi vacuoles increase by the 96th hour in the normal cell. Probably development of the Golgi complex parallels the transformation of rough ER (7). In the case of $f c$, the Golgi complex also develops normally until the 60th hour. At the 72nd or 84th hour, there is still the Golgi complex in the region where tubular or vesicular ER are observed, but in the region where lamellar ER predominates, the Golgi material is composed of small vacuoles or vesicules. The rough ER and Golgi complexes indicate that there is no augumentation of fibroin biosynthesis, as occur normally, in the $f c$ cell between 72 and $96 \mathrm{~h}$ (7). We have shown previously that $\left[{ }^{3} \mathrm{H}\right]$ glycine incorporation into silk gland proteins does not increase after the 72nd hour in the $f c$ larva, whereas in the normal silk gland the incorporation increases until the 120th hour (1). The cytoplasmic content of fibroin mRNA as assayed by the method of Suzuki and Brown (6) was low in the $f c$ posterior silk gland at $96 \mathrm{~h}$, but in the normal gland it was very high at the same stage (Maekawa, personal communication). This evidence suggests that the function of the fibroin synthesizing machinery is gradually impaired in the $f c$ glands.

The most prominent feature of the $f l c$ cell is the accumulation of large numbers of globules in the cytoplasm. The ultrastructural profiles of these globules (Figs. 1B and 2B) resemble what are called the "fibroin globules" that occur in normal silk gland cells (7). Moreovere, the apical cytoplasm of the $f c$ cell is similar to luminal fibroin when observed under a light microscope after staining Mallory's method (1). From these observations, together with the fact that the $f c$ glands contain fibroin like proteins, as detected by polyacrylamide gel electropholesis (Adachi unpublished), we have designated the globules in the $f l c$ cell fibroin globules. The accumulation of 


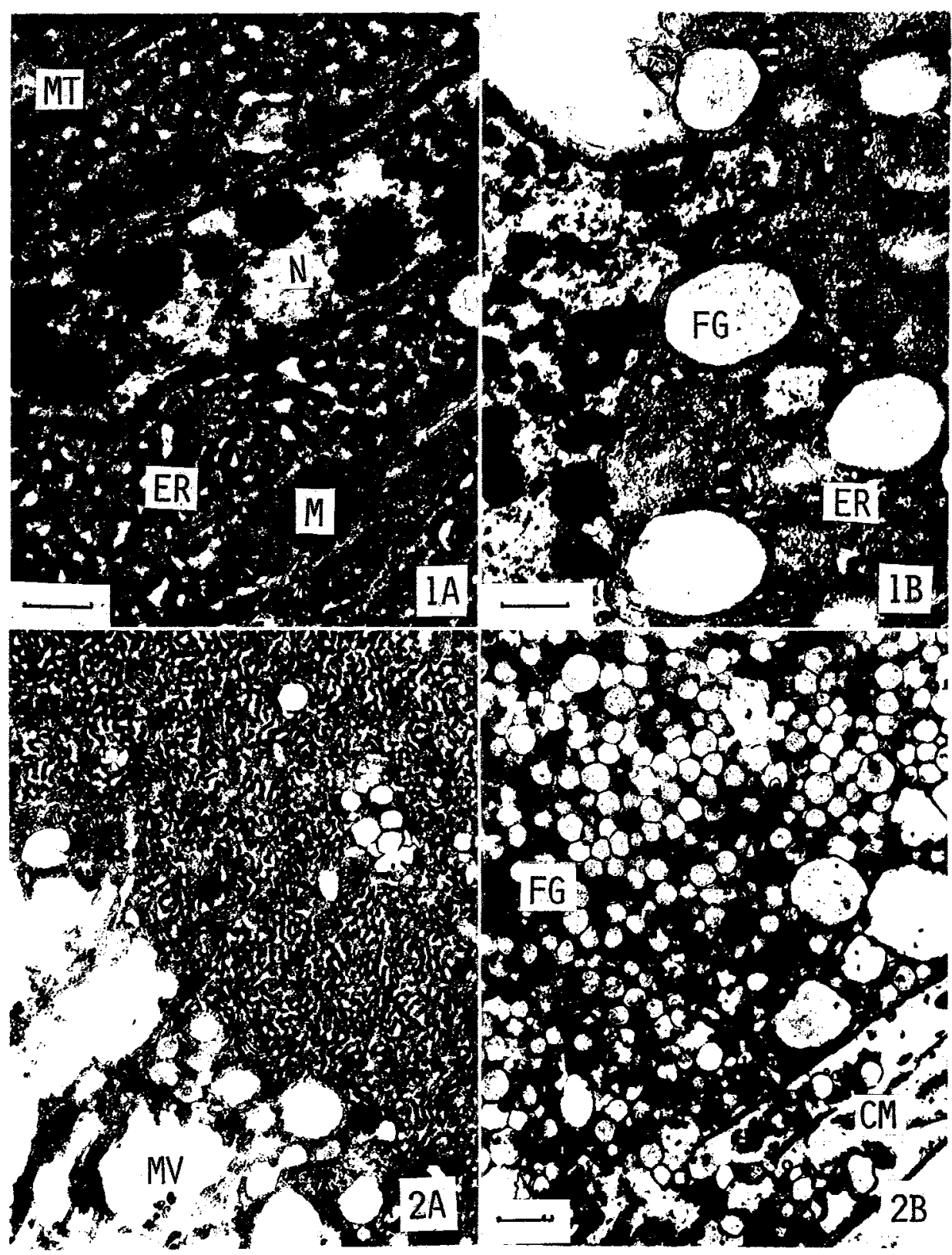

Fig. 1. Perinuclear region of the posterior silk gland cell at the 96 th hour of the fifth instar. A, normal cell: $\mathrm{B}, f l c$ cell. $\mathrm{N}$, nucleus: $\mathrm{ER}$, rough endoplasmic reticulum: $\mathrm{M}$, mitochondria: $\mathrm{MT}$, microtubules: FG, "fibroin globules" (see text). (Bar, $0.5 \mu \mathrm{m}$ ).

Fig. 2. Apical region of the posterior silk gland cell at the $96 \mathrm{th}$ hour of the fifth instar. A, normal cell: B, flc cell. MV, microvilli: CM, cytoplasmic masses in the luminal region: FG, "fibroin globules" (see text). (Bar, I $\mu \mathrm{m}$ ). 
globules first becoms evident at the 72nd hour in the apical cytoplasm (Fig. 2B). Nevertheless, fibroin synthesis may last at least until the 96th hour although its rate may be lower than normal. Thus the number of the globules continue to increase until they occupy the perinuclear region (Fig. 1B) and even the vicinity of basement membrane.

If the transport of the silk proteins in the mutant cell is inhibited at some step from ER via Golgi vacuoles to fibroin globules, then the intracisternal space of ER should be greatly distended, or the structure of Golgi vacuoles should change significantly, as has been reported for the posterior silk glands of the "Naked pupa" mutant of $B$. mori (4). In the case of $f l c$, no such changes in the ER and Golgi vacuoles could be detected, therefore, the $f c$ gene may not affect the intracellular transport of fibroin from ER to the Golgi vacuoles.

The accumulation of numerous secretory granules often is observed in the exocrine glands, as in the pancreatic exocrine cells of starved animals whose secretions are stimulated abruptly. In the case of these glands, the discharge of secretory proteins occurs at a limited rate without stimulus. When stimulated, in some cases, discharge proceeds in the absence of continuous protein synthesis $(2,5)$. Synthesis, transport and discharge in these glands appear to be controlled by separate mechanisms.

In normal posterior silk gland cells of the silkworm, the rate of fibroin discharge increases in proportion to the increase in the biosynthesis of fibroin. Thus the accumulation of secretory globules is relatively small in the normal cell. In contrast, the rate of fibroin discharge in the $f c$ cell may not increase in proportion to its biosynthetic rate after $72 \mathrm{~h}$. Therefore the secretion and synthesis of fibroin appear to be under independent control and its discharge might be facilitated by a product of the $+f l c$ gene.

In summary, we observed the accumulation of unusual numbers of globules which finally occupied the entire cytoplasm of the posterior silk gland cells of the flimsy cocoon $(f l c)$ mutant of B. mori.

Acknowledgements. We would like to thank Dr. H. Doira for his advice and for supplying the silkworms, Dr. K. Koga for his help in preparing the manuscript, and Professor R.C. King for reading the manuscript and for his valuable discussions. This work was supported in part by a grant from the Ministry of Education, Japan.

\section{REFERENCES}

1. Adachi, N. and H. Chixushi. Bio-histochemical analysis of gene action in the flimsy cocoon mutant of Bombyx mori. Sci. Bull. Fac. Agr., Kyushu Univ. 31, 159-173, 1977

2. Cuerdo-Rocha, S. and D. Zambrano. The action of protein synthesis inhibitors and thyrotropin releasing factor on the ultrastructure of rat thyrotrophs. J. Ultrastruct. Res. 48, 1-16, 1974

3. Dolra, H. Genetical studies of the flimsy cocoon of Bombyx mori. J. Sericult. Sci. Japan 42, $425-435,1973$

4. Inima, T. Ultrastructure of the posterior silk gland of the "Naked pupa" silkworm, Bombyx mori. J. Insect Physiol. 18, 2055-2063, 1972

5. PAlADE, G. Intracellular aspects of the process of protein synthesis. Science 189, 347-358, 1975

6. Suzuki, Y. and D.D. BROWN. Isolation and identification of the messenger RNA for silk fibroin from Bombyx mori. J. Mol. Biol. 63, 574-588, 1972

7. Tashiro, Y., T. Morimoto, S. Matsuura and S. Nagata. Studies on the posterior silk gland of the silkworm Bombyx mori. I. Growth of the posterior silk gland cells and biosynthesis of fibroin during the fifth larval instar. J. Cell Biol. 38, 574-588, 1968 